\title{
Wronski's Foundations of Mathematics
}

\author{
Journal Article
}

Author(s):

Wagner, Roy (i)

Publication date:

2016-09

Permanent link:

https://doi.org/10.3929/ethz-b-000120118

Rights / license:

In Copyright - Non-Commercial Use Permitted

Originally published in:

Science in Context 29(3), https://doi.org/10.1017/S0269889716000077 
Vol. 29, No. 3

\section{Wronski's Foundations of Mathematics}

Roi Wagner

ETH Zürich

E-mail: roi.wagner@mail.huji.ac.il

\section{Argument}

This paper reconstructs Wronski's philosophical foundations of mathematics. It uses his critique of Lagrange's algebraic analysis as a vignette to introduce the problems that he raised, and argues that these problems have not been properly appreciated by his contemporaries and subsequent commentators. The paper goes on to reconstruct Wronski's mathematical law of creation and his notions of theory and techne, in order to put his objections to Lagrange in their philosophical context. Finally, Wronski's proof of his universal law (the expansion of a given function by any series of functions) is reviewed in terms of the above reconstruction. I argue that Wronski's philosophical approach poses an alternative to the views of his contemporary mainstream mathematicians, which brings up the contingency of their choices, and bridges the foundational concerns of early modernity with those of the twentieth-century foundations crisis. I also argue that Wronski's views may be useful to contemporary philosophy of mathematical practice, if they are read against their metaphysical grain.

\section{Introduction}

Józef Maria Hoëné Wronski (1776-1853) was a Polish philosopher and scientist working mostly in France. He did not have much success in disseminating his ideas, and was marginalized by his contemporary scientific community. This paper will try to retrace Wronski's conception of mathematical foundations, and to explore the unique features of his philosophy of mathematics.

The motivation behind this reconstruction is not only the challenge of making sense of the ideas of a notoriously vague philosopher. The challenge is to reconstruct a path that mainstream mathematics did not choose to follow. Indeed, following the high road of the history of science, we usually confine ourselves to the tracks of successful scientists (either in their own lifetime or posthumously). This makes the chain of successful scientific developments appear natural and sometimes even necessary. It's only when considered against the roads not taken that we see that other courses of developments were possible, and that the actual evolution of science has a strong contingent dimension.

The history recounted in this paper is a history of such an abandoned road. Wronski, who did come up with some genuine and valid mathematical contributions, was primarily interested in reorganizing all mathematical knowledge around an elaborate post-Kantian philosophical system. Had he been successful, mathematics would have developed very differently. The current mainstream approach offers formal axiomatic frameworks such as the Zermello-Fraenkel axiomatization of set theory or the system of category theory. Any structure that can, in principle, be formally expressed in terms of these frameworks is mathematically legitimate (although not necessarily interesting). A valid proof is any argument where statements follow according to the relevant rules and toolboxes. But had Wronski's path been followed, mathematics would be derived from philosophical-ontological generative principles and unified under ur-statements purporting to 
subsume all mathematical knowledge. The legitimacy of mathematical entities and proofs would depend on identifying their position with respect to the generative ontological framework and the ability to derive them in accordance with the hierarchies of this framework. First principles and elementary entities would therefore have to fit this philosophical framework, which excludes many of the definitions and principles popular at the time. Wronski's mainstream contemporaries, however, did not endorse his approach. To fully appreciate the contingency of mainstream early nineteenth-century Parisian mathematics, we must understand which mathematical styles, projects, and priorities it rejected.

Reconstructing Wronski's path is far from easy. While he did hold on to a fixed philosophical ur-structure throughout his career, his articulations are often half baked and fluctuate across his oeuvre. His presentations often start "in the middle," supported by cross references to his other works, or they depend on justifications that he intended to introduce in subsequent works. On top of that, Wronski had a tendency toward esotericism, withholding some of his proofs and what he considered to be the deepest ideas of his philosophy, for which humanity was supposedly not yet ready (APOD, 2-3; PROL, 553-554). ${ }^{1}$

My task here is therefore to try and make sense of Wronski's philosophical-mathematical system. However, I will not try to "fill in the gaps" in Wronski's system so as to make it more coherent than I found it to be; nor will I evaluate Wronski's system based on subsequent mathematical developments. Instead, I will try to come up with an interpretation that highlights Wronski's main schemes of thought, those rejected by his contemporary scientific establishment. As an aside, I'll hint at a certain (ab)use of Wronski's ideas that might be relevant for contemporary philosophy of mathematical practice. The analysis brought here complements a more socially embedded analysis of Wronski's ideas around the use of actual infinities in Wagner (2014), which also includes a biographical sketch and an overview of his mathematical contributions.

In order to probe into Wronski's work, I'll start with the way many of Wronski's contemporaries first came to know him: through his critique of Lagrange's algebraic approach. After extracting the main distinct points of his critique, I'll try to put them together into his broader philosophical framework. Then, to make things more concrete, I'll show how this framework plays out in proving his most prominent result: the "universal law" for expanding any function in terms of any sequence of functions.

\section{Very Brief Background on Wronski}

Jozef Hoene (later Hoëné Wronski) was born near Poznan to a Czech architect migrant. He enlisted into the Polish army, was captured by the Russians, and ended up having a short but successful career in the army of his former enemies. He then traveled to study in Germany, and in 1800 settled in Marseille. During a decade of work he created a post-Kantian philosophical framework and engaged in mathematical and astronomical research. In 1810 he felt he was ready to move to Paris and expose his mathematical work to the Académie des Sciences.

His first submission to the Académie (later published in INT) received a reserved but generally supportive critique from Lacroix and Lagrange, who were impressed by the generality of Wronski's result (which was a version of the universal law given below). They scolded him, 
however, for vague ideas and missing proofs. Wronski did not think that the critique did him justice, and entered into public polemics with various prominent mathematicians. He was quickly marginalized to the point of barely being able to support himself financially. A rich patron-student came to his aid, but within three years a financial controversy turned their relationship into a highly publicized scandal, which involved printed allegations concerning unpaid debts, abuse of trust, the disclosure of the secret of the absolute, and clandestine satanic societies.

Wronski went on to try his luck in London and Belgium, but managed to antagonize scientists there as well. He moved on to publish his ideas on political philosophy while trying to advance various inventions, including logarithmic tables, a calculating machine, and some sort of running track-chains to replace railways (as in modern military tanks). Finding little success, Wronski survived due to the support of a small circle of followers impressed by his transcendent philosophy and doctrine of "messianism": a rational union of philosophy, religion, liberalism, and authoritarianism, which was to lead humanity to unity with the absolute.

Wronski's mathematical work revolved around series expansions and solutions to polynomial, congruence, and differential equations. Wronski's most notable mathematical contribution is his "Supreme Law," whose proof is reviewed below: a formula for the coefficients of the development of any function $F$ by any sequence of functions $\Omega_{1}, \Omega_{2}, \Omega_{3}, \ldots$. He delved into various transformations and efficient choices of $\Omega_{i}$ in order to induce or improve convergence. In the context of differential equations, he constructed homotopies between given equations and linear equations with constant coefficients; then, starting from the known solution of the linear equation, one expands the solution of the original equation as a power series of the homotopy parameter (see West 1886). In the context of polynomial equations, he suggested an algebraic method for reducing their degrees in order to prove (the false claim) that polynomial equations have solutions in terms of roots of their coefficients; the supposed reduction, however, would often increase the degree of the equation. But Wronski also devised other successful methods for reducing and solving polynomial equations based on series expansions and on reiterated substitutions (REFIII), drawing on his own work and on that of Daniel Bernoulli, respectively. His efforts in the context of congruence equations of the form $x^{m}=a(\bmod b)$ extended Gauss' solution of linear congruence equations, and aim at reducing the range of searching for solutions, rather than actually providing them (REFI). Another achievement was Wronski's compact logarithm tables published in 1827 (CAN). These were based on the first terms of a series expansion of the logarithm. The trick was to give, instead of the full logarithm, a simple way to pick up from a single compact sheet of paper two or three blocks of digits that compose the desired logarithm (for elaborations and bibliography related to these contributions and their later evaluations, see the appendix in Wagner 2014).

In many ways his mathematics was in line with eighteenth-century Eulerian mathematics, and failed to live up to the standards of later nineteenth-century analysis of convergence. He made many exaggerated claims to have solved all major mathematical problems, and committed quite a few mathematical errors, but he also made some important mathematical contributions, which, due to his marginalization, surfaced only when they had lost their innovative value, in the last three decades of the nineteenth century.

Wronski's philosophy attempted to build on Kant's, and then advance beyond Kant to an analysis of the transcendent absolute. He was influenced by German idealism, and commented on the leading contemporary German thinkers (Fichte, Schelling, Hegel). His philosophical system was 
mostly classificatory in nature: positing a certain ur-structure, he attempted to fit all philosophical and scientific knowledge into this structure. At the foundation of this structure lay alleged esoteric insights into the intimate nature of the absolute, which Wronski refused to disclose.

Wronski left behind a large collection of thick publications and manuscripts, which, however, attracted only relatively little interest after his death. ${ }^{2}$ Brief biographies and summaries of Wronski's mathematics are included in Phili (1996), Murawski (2005), Pragacz (2008), and Wagner (2014). A full biography is available only in Polish (Dickstein 1896). The best surveys of Wronski's mathematics are Dickstein's (1892-1896) brief outline and West's (1886) more detailed elaboration. His philosophy is surveyed comprehensively in Warrain (1933-38) and d'Arcy (1970), and summarily in Murawski (2006).

\section{Wronski vs. Lagrange}

In order to present Wronski's ideas in their original context as first exposed to his contemporaries, we'll review Wronski's objections to Lagrange's algebraic presentation of derivatives as coefficients in Taylor series. I believe that the interpretations of these objections by his contemporaries and by later scholars have not captured Wronski's main contentions, so a detailed review is required here.

Lagrange's approach, in a nutshell, is this. Given what was then called an analytic function $F$ (that is, a function given implicitly or explicitly in terms of elementary analytic functions and arithmetic operations, see Medvedev 1991, 32-49), and a fixed point $x$, the function $F$ can generically be written as

(1) $F(x+i)=F(x)+i P(x, i)$.

Lagrange defines the derivative of $F$ at the point $x$ to be the value $p=P(x, 0)$, and denotes it $F^{\prime}(x)$. Then we can reiterate the same construction for $P(x, i)=p+i Q(x, \mathrm{i})$, and continue so as to obtain a power series:

(2) $F(x+i)=F(x)+p i+q i^{2}+r i^{3}+\ldots$.

Using the above definition of the derivative, and equating the expansion of $F(x+i+o)$ around $x$ with its expansion around $x+i$ (further expanding $P(x+i, o), Q(x+i, o), R(x+i, o), \ldots$ around $x$ ), the coefficients $p, q, r, \ldots$ turn out to be the coefficients of the Taylor series: $F^{\prime}(x), F^{\prime \prime}(x) / 2 !, F^{\prime \prime \prime}(x) / 3 !, \ldots$, where multiple dashes represent iterations of Lagrange's definition of the derivative (Lagrange 1797, 13-14).

Lagrange did acknowledge that for $P(0)$ to be finite, sometimes, instead of $i$, a fractional power of $i$ should be used. However, he argued that this can happen only at isolated points, as otherwise the fractional powers in the series expansion would force their multivaluedness on the original function (note the global view of functions here, typical of the contemporary mathematical mindset, which preferred not to reduce a multivalued analytic function to just one of its branches). Lagrange also acknowledged that the convergence of the power series could not be taken for granted. But while he believed that the power series represented the function even where convergence failed (Lagrange 1797, 67), he also proposed a bound for the error when truncating the series after its first $n$ terms, in order to guarantee the meaningfulness of the series representation in finite terms. 
Wronski, on the other hand, insisted that the only correct way to view the Taylor series was as a special case of his universal law (presented already in INT, but proven only in TEC). ${ }^{3}$

(3) $F=a_{1} \Omega_{1}+a_{2} \Omega_{2}+a_{3} \Omega_{3}+\ldots$.

This law (whose validation will be discussed in section 7 below) presented formulas for the coefficients of the expansion of any function $F$ in terms of any sequence of functions $\Omega_{i}$. The coefficient formulas were based on "combinatorial sums" (in modern terms: determinants) of differences or differentials of $F$ and the $\Omega$ 's (LAG, 15-17). ${ }^{4}$ For Wronski, the differential was an infinitesimal difference, and actual infinitesimal quantities were necessary elementary mathematical objects, even though they existed in reason, and not in empirical experience (for details, see Wagner 2014).

Wronski reacted to Lagrange's approach with several technical and philosophical objections, which were meshed up together in LAG. The technical objections seemed to his contemporaries as either weak or already resolved, and the philosophical objections depended on a framework that was not clearly set. Therefore, Wronski's arguments were dismissed by most readers, including Legendre and Arago, who, on November 11, 1811, wrote a condemning report to the Académie des Sciences on a manuscript of the first part of LAG. I'll open the discussion of Wronski's ideas by trying to articulate Wronski's technical and philosophical objections under three distinct headings.

\subsection{Universality vs. Individuality}

Wronski's first point was that the expansion of a function could include fractional powers. To answer Lagrange's objection that this would entail multiple values, Wronski claimed that the different branches of fractional powers in the series could all eventually sum up to a single value for the resulting function (LAG, 22). As an example, Wronski considered the expansion of a function in terms of the sequence $1,\left(a+x^{1 / m}\right),\left(a+x^{1 / m}\right)^{2},\left(a+x^{1 / m}\right)^{3}, \ldots$ according to his universal law. He claimed (without the support of a calculation or other arguments) that the coefficients are such that the fractional powers would combine to produce a single end result.

Later on, in a section written after he had received Legendre's and Arago's dismissive critique for the first part of the essay, Wronski qualified his argument more carefully:

considering the function $f(z+h)$ in its numerical result, it is true that it will only contain integer powers of $h$, but this particular circumstance is purely contingent and does not apply necessarily to the very nature of the expansion. ... The coefficients [of fractional powers in the expansion,] which here turn out to be zero, exist really [as coefficients in the universal law (3)], and have determinate expressions [general formulas that determine their value according to the universal law]. (LAG 50)

Wronski even used Lagrange's own multi-valuedness argument to explain why the coefficients of fractional powers would turn out to be zero; but he nevertheless insisted that these coefficients do exist, and that their null value is not in the "nature" of the expansion, but only a secondary circumstance (that said, Wronski never attempted an expansion of a function in terms of a series of

3 According to Wronski, from one point of view (theory) this was the "supreme law," and from another (techne) it was the "universal law." I use the latter throughout. The distinction between theory and techne will be presented below.

4 These coefficients are closely related to what Muir (1882) later called the Wronskian matrix - a term in use to this very day. 
all fractional powers of the variable; he had some implicit judgment as to which powers or algebraic functions were relevant for a given expansion).

In a subsequent attempt to explain this contingency, Wronski began with the well known expansion

$\log (x)=(x-1)-\frac{1}{2}(x-1)^{2}+\frac{1}{3}(x-1)^{3}+\ldots$,

which, substituting $z^{1 / m}$ for $x$, becomes

$\log (z)=m\left(\left(z^{1 / m}-1\right)-\frac{1}{2}\left(z^{1 / m}-1\right)^{2}+\frac{1}{3}\left(z^{1 / m}-1\right)^{3}+\ldots\right)$.

Now, using the binomial formula and rearranging so as to construct a series expansion in terms of $z^{i / m}$, the coefficients turn out to be infinite, rather than zero:

$\log (z)=-m\left(\infty-\infty z^{1 / m}+\frac{\infty^{2}}{2} z^{2 / m}-\frac{\infty^{3}}{3} z^{3 / m}+\ldots\right)$ (LAG, 97-103; the different powers of infinity represent the fact that each infinite coefficient is the sum of a different divergent series).

It should be obvious by now that Wronski is using a language different from ours and from that of his contemporaries. What does it mean that the coefficients must be zero, but that this nullity is not in their "nature"? What sense is there in a power series with infinite coefficients?

To make sense of this, we must recognize the different layers in Wronski's conception of mathematics. There's the layer of the universal law, where all functions are subsumed under general analytic formulas; then there's the individual layer of actual values substituted into these formulas, which may yield null or infinite values. The fact that the coefficients of $z^{i / m}$ are expressible by the formulas of the universal law means that these coefficients cannot be ignored, even if, when we calculate them through, they turn out to be infinite or null. Reason can legitimately consider the generation of coefficients (how they are formed analytically from elementary quantities and operations), even where these coefficients are not present in empirical experience because they turn out null or infinite.

A distinction between the formula and its values may seem objectionable at first sight, due to the modern definition of a function as consisting of ordered pairs of its arguments and values. But I would like to suggest that this distinction reflects a matter of practice. Indeed, the formula as a combinatorial object is a (meta)mathematical object in its own right. For example, it is this very kind of distinction between formulas and values that allowed later mathematicians to abstract terms such as $x y-y x$ from their null value, so as to enable non commutative re-interpretations. But Wronski's conceptualization doesn't stop there. It overdetermines the practical difference between formulas and their values by a philosophical difference. As will be explained below, he argues that formulas and their values are not reducible to each other, and carry different epistemological purport. They reflect the capacities of different intellectual faculties and different aspects of knowledge.

\subsection{Possibility vs. Necessity}

Wronski's next set of objections concerned the very assumption that a function should be expandable as a power series, regardless of whether these powers are integer or fractional. Wronski considered Lagrange's assumption, $F(x+i)=F(x)+i P(x, i)$, as unfounded, because no argument was provided to validate it beyond the obvious identity at $i=0$ (LAG, 23). This, in turn, casts a shadow 
on the entire derivation of the series (2).

The doubt concerning the possibility of this form of expansion predates Wronski, and one notable attempt to justify it was offered by Poisson. Poisson (1805) proved that if a function could be written as

(4) $F(x+i)=F(x)+i^{a} P(x)+i^{b} Q(x)+i^{c} R(x)+\ldots$,

then the powers $a, b, c, \ldots$ must be positive integers. ${ }^{5}$ Wronski conceded that under the assumption of the expansion (4), all powers with nonzero coefficients do turn out to be integer powers, but questioned the grounds upon which the expansion (4) was considered legitimate in the first place (LAG, 44-45, 53).

One should be careful not to confuse Wronski's critique with one based on a modern notion of function, where the assumption of the expansion (4) is indeed restrictive. Wronski did not reject the possibility of the expansion, he only demanded that it be proved. We see here that for Wronski the very comparability of two different mathematical forms - a given analytic function and an infinite expansion - is problematic in itself. This is not because Wronski had a richer concept of function, but because he considered analytic functions and series as different kinds of mathematical entities belonging to two different mathematical levels: an analytic expression captures the nature of a function as composed of elementary analytic entities and operations, whereas a series captures the generation of the values of a function (the former belongs to theory, and the latter to techne; we'll explain this distinction later on). Unlike modern mathematicians, Wronski did not reduce infinitary expressions into finitary ones (by force of limit-like definitions), but considered them as ontologically elementary entities. Accordingly, a comparison of finitary and infinitary entities requires justification.

In fact, Wronski did acknowledge as legitimate a process of forming the Taylor series through step-by-step reiteration, akin to that of Lagrange above, without going through his universal law (3) - but this was considered well founded only after the possibility of this evaluation had been granted: "in postulating the possibility of such an evaluation [referring to a generalized variant of $F(x+i)=F(x)+i P(x, i)$ ], this [Lagrange-like] deduction ... reduces manifestly to establishing its NECESSITY" (TECI, 44). Wronski distinguishes here between two components of proof: the component of possibility, where the formula such as (1) is legitimized, and the component of necessity, where, given this possibility, the manipulation of the equated forms lead to the necessarily resulting values.

It seems that the use of the dichotomy possibility/necessity is not consistent throughout Wronski's work, which makes interpretation problematic. It would help, therefore, to correlate this dichotomy with another, as in the following statement: "In the technical [stepwise, Lagrange-like] deduction, only the conception of FINALITY comes in ... in the theoretical deduction [of the legitimacy of the equation] we required a SPECULATIVE principle" (TECI, 84). "Speculative" here relates to reasoning with concepts. "Finality," on the other hand, has to do with constructive acts of will that generate something new in the world. Speculative reason establishes the possibility of the expansion by conceptually legitimating the comparison of the two different kinds of entities. Then, the finality inherent in iterative acts of will (Lagrange's reiterations) constructs the expansion

5 Poisson equated two expansions of $F(x+2 i)$ : the first around $x$, and the second around $x+i$ (with a further expansion of the coefficients $P(x+i, i), Q(x+i, i), R(x+i, i), \ldots$ around $x)$. He found that for the lowest power $a$ one has $(2 i)^{a} P(x)=2 i^{a} P(x)$. Hence, for nonzero $P(x)$ we get $a=1$. Then one can go on and argue concerning the higher powers. An implicit assumption here is that the powers in the expansion do not change when moving from $x$ to $x+i$. 
step-by-step, and establishes what necessarily derives from the repeated evaluation. ${ }^{6}$

This possibility/necessity or speculation/finality distinction has to do with the distinction between proving deductively the existence of a certain kind of solution and a heuristic postulation of this existence so as to calculate the solution. However, Wronski's conception of the former is significantly different from the contemporary one, as it is metaphysical, rather than merely mathematical. A proof of the possibility of an equation between two different ontological kinds (such as a closed analytic formula and an infinite power series) must account for the ontological maneuver that turns one into another (Wronski's account is presented in section 7.1).

The suspicion raised against comparing the two kinds of mathematical entities (analytic functions and infinite series) may appear unwarranted to us, as in modern terms these are just two representations of mapping numbers to numbers. But the qualitative distinction between different forms of representation is in fact part of mathematical practice, and was even more significant under the influence of Euler, in a mathematical culture where series were not necessarily reducible to their values (Ferraro 2007).

In more general terms, the caution against comparing different kinds of mathematical entities has a long history in mathematical practice: in various times and places, the comparability of non homogeneous quantities was and still is questionable: this was not just the view of purist classical Greek geometers, who were careful not to mix, say, numbers and geometrical magnitudes, but also part of the practice of contemporary applied mathematicians, who take care to make sure that the units and scales on either side of an equation balance out. A reflection on the legitimacy of certain kinds of comparisons is an important part of mathematical practice. But Wronski overdetermines this practical reflection with a philosophical distinction between the acts of different faculties (understanding and will) that we shall elaborate below.

\subsection{Form vs. Meaning}

The two kinds of objections above concerned the legitimacy of the form of the expansion (2). But Wronski takes care to emphasize that this is only an accessory concern (LAG, 62, 66). Even if this form were given (and indeed, Wronski was going to establish its "possibility"), Lagrange would still be missing the main point. Indeed, in Lagrange's method, according to Wronski, "the derived functions $F^{\prime} x, F^{\prime \prime} x, F^{\prime \prime \prime} x$ etc. necessarily remain blind functions, or at least cyclops functions, that is functions that have only a name and no signification. And it is this signification that we propose to discover" (LAG, 26). To support this claim, Wronski argues that if derivatives had originally been defined (before the onset of infinitesimal calculus) as they were by Lagrange, namely as coefficients in an expansion, they would have been viewed as an "algorithmic mystery" (LAG, 25).

In what does the missing "meaning" of the coefficients consist? For Wronski the criterion is clear: "the combination of algorithmic procedures or operations that come together in the generation of a quantity constitutes the nature, and therefore, for us, the signification of that quantity" (LAG, 69). The signification of a quantity lies in its construction from elementary quantities and arithmetical operations. For Wronski, neither Lagrange's description of the formal relations between differentials and their primitive functions by means of Taylor series, nor his calculation of their

6 A similar contrast appears in various other contexts in Wronski's work, such as the discussion of the fundamental laws of number theory as opposed to the construction of solutions of congruence equations (REFI, 26, 75). 
values in specific cases, is enough to render them meaningful. What's missing is an independent substantive construction of differentials

To provide the meaning of differentials, one would need to provide them with generative formulas. These formulas were given by Wronski for the most general case of the universal law (3) (see the proof of the universal law in section 7). In the special case of the Taylor or power series (2), infinitesimals must be substituted into the formulas of the coefficients of (3) and eventually, after simplification, come down to the definition of derivatives as quotients of infinitesimal differentials

- the well known $\frac{d f}{d x}=\frac{f(x+d x)-f(x)}{d x}$, where $d x$ is an infinitely small quantity. This is the definition that, for Wronski, captures the nature of derivatives in terms of elementary mathematical entities and operations, and is therefore the only philosophically viable definition.

We see here a constructivist tendency in Wronski's work, but it's important not to confuse it with modern forms of constructivism or intuitionism. Indeed, unlike modern constructivists, Wronski embraced the use of actual infinity. In fact, he vehemently refused to displace actual infinities from their position as elementary and constitutive entities in his system.

For example, Wronski showed how to derive the coefficients of the Taylor series (that is, derivatives of a function $F$ ) from the coefficients $A_{i}$ of the series $F(x)=A_{0}+A_{1} x+A_{2} x(x-\xi)+A_{2} x(x-\xi)(x-2 \xi)+\ldots$, and pointed out that the formulas for the $A_{\mathrm{i}}$ 's derived from the universal law (3) did not involve infinitesimals (but did involve infinite sums). ${ }^{7}$ However, this construction of the derivatives of $F$ required the whole infinite series of $A_{i}$ 's, and therefore did not construct derivatives as finite combinations of elementary quantities and operations. Instead, Wronski proposed that the nature of derivatives was correctly captured only by their direct determination as quotients of the infinitesimals $d f$ and $d x$ (a finite combination of subtraction, division, and infinitesimals). In other words, the former construction gave the means to calculate the values of the derivatives via the indefinite construction of $A_{i}^{\prime}$ 's, but only the latter construction could determine their essences as finite combination of elementary quantities and operations. A finite combination of actual infinitesimals was considered more fundamental than an indefinite combination of finite values (INF, 23-25, 58, 83-87).

As in the previous two subsections, this approach reflects a difference in mathematical practice: formal definitions from relations, axioms, and recursion, as opposed to direct finite constructions from given elementary entities. Constructive and formalist practices both take part in shaping mathematical knowledge, and are recognized as contributing to different forms of understanding. We don't have to take Wronski's side here (that is, prefer finite combination of actual infinities to indefinite combinations of finite terms), in order to acknowledge that the distinctions form/substance or immediate construction/indefinite expansion play a part in the production of insights by mathematical practitioners. But Wronski's philosophy overdetermines this distinction with an architecture that relates the finite and the infinite as expounded below.

\section{Wronski's Philosophical Foundations}

The above analysis covers a set of distinctions that Wronski's contemporaries either did not 
appreciate or failed to understand. In this section I'll begin systematizing Wronski's conception of mathematical foundations so as to put the above objections in their philosophical context. We'll start from some general points.

The first point that needs to be considered is the link between mathematics and philosophy. According to Wronski, "the metaphysics or the deduction of the first principles of differential calculus is entirely beyond the limits of mathematics; as a consequence, it is a plain waste of effort when mathematicians try to deduce these principles by purely mathematical means. It is to a higher order, to philosophy, that this task belongs" (LAG, 36-37).

Wronski did not consider himself a mathematician, but a philosopher of mathematics, and therefore not in competition with mathematicians (INF, 183). He believed, however, that his discoveries laid the foundations that mathematicians should work with, and often digressed into mathematical calculations so as to demonstrate how his philosophical principles enabled the supposed solution of all major mathematical problems.

French mathematicians who dabbled in philosophy, according to Wronski, tended to fall prey to a materialist or empiricist philosophy (LAG, 54; LAP, 2) of the kind associated with d'Alembert, Condillac or Condorcet: an attempt to prioritize empirical experience and view the constructs of reason as linguistic aids or as abstractions that should be related back to worldly experience (Wagner 2014).

Wronski's own philosophy depended on Kant's transcendental philosophy, which explains how experience is constrained by the knowing subject (an analysis of the mind's categories and forms that shape our knowledge). However, as early as Wronski's first attempt at a philosophical treatise (PHI), he already announced that his philosophy would reach farther than Kant's, into the founding transcendent, infinite, and absolute principles that condition the knowing subject and the known object alike. Here he follows the path of German idealist philosophers (such as Fichte, Schelling, and Hegel) who took upon themselves to unite that which Kant had set apart - the knowing subject and the known world. While Wronski remains faithful to the basic Kantian terminology, his use of these terms is often rather idiosyncratic or influenced by its later appropriations by German idealists. The Kantian kernel of his thought lies in the discrepancy between finite and constrained receptivity of sense data on the one hand and open ended spontaneous reason on the other; the post-Kantian kernel of his thought is the belief that this discrepancy can be subsumed under an absolute that is accessible to the human mind.

Being a philosopher who intends to account not only for the transcendental (the conditions that shape human knowledge), but also for transcendent truths and entities (independent of human knowledge), Wronski believed that philosophy must reduce its objects of study to absolute and independent philosophical principles (REFII, 108). Mathematicians such as Kramp and Arbogast were therefore criticized for dealing with a host of mathematical identities without a unifying law (TECII, 106), and Lagrange was criticized for introducing the two secondary principles (1) and (2) for his calculus instead of a single fundamental principle (LAG, 23). The fault of lacking a unique foundation was attributed to Laplace's theory of generating functions (1812) as well (LAP, 120; TECI, 153), but Laplace was credited with having at least felt the need for a unique founding principle, even if he could not supply it (REFII, xcvii). Wronski himself, on the other hand, pretended to base all mathematical equations on his single universal law (3) (LAG, 17; TEC 141143), and reduce all problems to a single universal problem (expanding an implicit function into a series, see TECI, 169-171; REFI, 73). 
Now, in order to establish the fundamental mathematical law, its possibility (namely, its legitimation as a statement that could make sense) must be derived philosophically from first principles. Once this possibility is philosophically established as a founding synthetic principle, all that should remain would be to reduce it to a pure logical identity, independent of all other mathematical statements. Indeed, given their possibility, the formulas for coefficients in Wronski's universal law (3) were said to be reducible to the single analytic identity $A=A$ (LAG, 134; TECII, 14). This logical reduction should not be confused with modern logicism or formalism, since Wronski insisted on constructing a philosophical-synthetic scaffolding for the framework in which analytic reductions should take place; in his view, without such scaffolding mathematics would be an empty abstraction that ignored the nature and meaning of mathematical entities.

Wronski's demand for a single solid foundation should not be taken for granted. Indeed, during the first couple of decades of the nineteenth century, the French mathematical establishment wavered between several approaches. One was the Lagrangian algebraic analysis, which introduced the elements of analysis through formal-algebraic (and more or less finitist) means, such as the definition of derivatives as coefficients in a power series. The second, identified with d'Alembert, had a more geometric flavor, and tried to articulate analytic entities through limits in order to hold on to a tangible intuitive basis. The third approach did not shy away from using actual differentials and infinitesimals, even though they were subject to the danger of running into contradictions (see Schubring 2005, chap. 4, for a comprehensive review).

But not everyone took sides. Many treated the whole problem opportunistically, and thought that the different approaches were interchangeable. Lagrange, who advocated the algebraic approach, did not try to enforce it in his work on mechanics. Carnot ([1813] 1832, 127-128) explicitly suggested that any approach was as good as any other. Cauchy's ([1821] 2009) approach, which is usually presented as the triumph of limits, was actually a compromise between limits and infinitesimals (while infinitesimals were defined in terms of limits, Cauchy's actual practice shows that this reduction did not exhaust his conception; see Schubring 2005, chap. 5). By 1812 the polytechnic had decided that analysis should be taught in terms of infinitesimals, and so Wronski could claim that Lagrange's approach was no longer taught in schools (TECII, 645). However, Lagrange's approach did survive in Wronski's lifetime through mathematicians such as Arbogast and Servois, and through the later proponents of Hindenburg's combinatorial school.

Another contemporary foundational controversy revolved around the relations between algebra and geometry. Geometry enjoyed a long established status as true and well founded, whereas algebra and analysis were newer and more suspect. As a result, notions such as negative and complex numbers were often seen as problematic. But the algebraic approach to analysis led to attempts to found algebra independently of geometry, including, among others, that of Bolzano, who sought to divorce the proofs of the intermediary value theorem and of the fundamental theorem of algebra from geometry. Wronski's approach took things one step further. He thought that trigonometric functions, for example, should be defined in analytic terms, as sums of complex exponents, rather than geometrically, through circle representations (INT, 17-19, 24-26). Later on (PROL, 221), true to his "single foundation" approach, Wronski went back and revised his earlier (cursory) treatment of geometry in INT, claiming that geometry is reducible to infinitary algebraic analysis.

An approach which had some affinity with Wronski's conception of foundations is that of Hindenburg's combinatorial school (Séguin 2005; Jahnke 1993). Like Wronski, this school had a main fundamental theorem: the polynomial theorem, which extended the binomial theorem to a 
multinomial or infinite sum. A reduction of the polynomial theorem to the binomial one is not too hard, but was not considered as proper foundation; as in Wronski's case, the correct foundation had to consist of a reduction of theorems to the bare elements independent of all other mathematical facts, that is, to combinations of summations and multiplications (Jahnke 1990, 191). ${ }^{8}$

But a more profound philosophical similarity between Wronski and Hindenburg's combinatorial school is harder to establish. Fries, a philosopher closely tied to Hindenburg's school, argued that mathematics is the creative imagination's free handling of the concepts of pure reason (ibid., 228-229). This echoes some conceptions of Fichte, an influence which Wronski acknowledges as well. Indeed, Wronski would gladly welcome accounts of pure reason as a source of mathematical objects (such as absolute infinity); however, he would reject the creative imagination as having anything to do with it (if we are to judge from INT, 167). He would also strongly reject Hindenburg's proto-formalist statements, such as his claim that mathematics deals with elements abstracted from their roles and meanings, and becomes relevant to reality only through an empirical application of these empty schemes to real entities (Jahnke 1990, 119).

So Wronski believed that mathematics should be based on a single universal law reduced to basic philosophical principles and logical identities. But we also saw in the critique of Lagrange that Wronski's mathematical world is broken into various components of mathematical ontology (or, if we read him against the grain, mathematical practice): universal formulas vs. individual values; mathematical elements that exist in reason (e.g. infinitesimals) vs. those that exist in experience; definite vs. indefinite mathematical constructions (analytic functions vs. series) that cannot be compared before the possibility of the comparison is justified; and empty formal definitions vs. substantial constructive ones. Wronski's philosophy of mathematics should therefore account for these distinctions and, at the same time, be able to unify them all under a single universal principle.

\section{Wronski's Law of Creation and His Notion of Theory}

We can now move on to the general structure in terms of which Wronski accounts for his unification of the diverse aspects of mathematics. In this presentation, I will only point out those elements of Wronski's system that are required to understand his mathematical moves, and will not attempt an overall philosophical analysis of the kind available in d'Arcy (1970) and Warrain (1933-1938)

The starting point of Wronski's philosophical system (viewed synthetically, that is, from the foundation up) is the absolute. Now, one could hold the view that reason is too limited to reveal the absolute essence of things (at least not without the aid of divine revelation), and therefore that the absolute is outside the reach of philosophy. But for Wronski this would be a self contradictory claim.

8 The hierarchical nature of the combinatorial school's proof system is witnessed by considering the inversion theorem (which provides the coefficients for the power series expansion of the inverse of a power series) as more fundamental than Lagrange's theorem for the power series expansion of an implicit function, and so the former could legitimately prove the latter, but not vice versa (Jahnke 1990, 202, 205). Modern mathematicians would embrace either approach, but Wronski too forced an order on the derivation of theorems that from a modern point of view are equivalent. We also note that for Hindenburg and his colleagues, closed formulas composed of elementary mathematical entities and operations were of the utmost importance, as they were considered more fundamental than recursive ones (ibid., 189-190). The theme of deriving closed formulas is consistently present in Wronski's work (e.g. TECII, 30, 5862; REFI, 123, 307), but, recognizing the computational advantages of recursive formulas, Wronski took care to derive the latter as well (e.g. TECII, 136-140; REFI, 317). On top of these formal similarities, the influence of Hindenburg's school on Wronski is witnessed by direct references to Hindenburg (e.g. TECII, 36, 76, 539) and to related 
How, in fact, can you recognize the incapacity of reason except by the faculty of your reason? You agree that it has the power to discern its own incapacity. And how can you conceive of this superior capacity of reason except by founding it on the very faculty of recognizing the absolute or the principle of things? In fact, if reason had not had this high faculty, it would not be able to doubt itself. (REFII 510-511)

From this self sufficiency of reason the definition of the absolute can be gleaned. The absolute is "the first principle, which contains in itself the reason of its own existence, that is, the condition of its proper reality, and which subsists thus in itself; a principle which, consequently, carries in it the condition for the reality of the universe" (REFII 512-513; see also Warrain 1933, 33; Fichte's influence is evident here).

This absolute has two distinct determinations: its creativity as self genesis, which is termed knowledge (but does not necessarily belong to a subject), and its permanent fixity and inalterability, which Wronski calls being. The spontaneity of knowledge and the inertia of being are neutralized in the intermediary reality of the absolute (APOD, 5-6). Moreover, according to Wronski, the unconditional principle to which everything can be reduced is Schelling's principle of the identity of knowledge and being - an identity between free creativity and the actual created world. Indeed, if there hadn't been such an identity, we'd have two separate lived systems, rather than a single reality (APOD, 5; NOM, 117; REFI, 49).

Wronski's entire philosophy can be seen as a rigid structure of distinctions and mediations deriving from this threefold synthesis, which eventually lead to a final unification. This structure is formalized in a complex recipe called "the law of creation." In fact, all systems in the world are constructed according to the same recipe, so we end up with a system homological to its subsystems - a sort of fractal that reiterates the law of creation (REFI, 63-64). It would be far beyond the scope of this essay to analyze the abstract articulations of the structural recipe of the law of creation, but we can quickly review the specification it receives in the context of mathematics.

The first primordial elements of mathematics are summation and "graduation," the latter referring to powers and roots (INT, 6-10). Summation is the discrete and finite collection of elements, which Wronski associates with the Kantian faculty of understanding (the faculty in charge of handling the concepts to be applied to finitary sense perception). It is on the side of the inert, given being. Graduation, on the other hand, depends on continuity, which, in turn, is viewed not as an objective sense datum, but as part of the way the world is known and regulated by the Kantian faculty of reason (Vernunft), which can form and relate concepts unbound to sensible intuitions. As graduation stands in opposition to the finite discreteness of summation, it has to do not only with continuity, but also with infinity: Wronski understands continuity as generated by infinitesimal change (REFI, 13). Multiplication is cast in the role of the primordial element that "neutralizes" summation and graduation (just as reality neutralizes being and knowledge), providing these distinct elements with a common liaison. Wronski associates it with the Kantian faculty of judgment, which connects reason and the understanding by rendering the formalisms of reason accessible to the understanding in a manner that allows them to be applied to sensible intuitions.

All this probably sounds quite odd to a modern reader (as well as to modern Kant scholars). I can reassure this modern reader that it sounded odd to Wronski's contemporaries as well. In fact, even a reader who, unlike most of Wronski's contemporary French readers, was versed in Kantian philosophy and the nascent German idealism, could probably not make too much sense of it. Wronski's argumentation is scant. For example, it fails to explain how multiplication "neutralizes" 
the antagonism between summation and graduation, beyond the fact that one may think of integer multiplication as many summations, and of integer powers as many multiplications.

Wronski's INT goes on to apply the recipe of the law of creation (a closed schematic system of combinations, transitions, influences, and identifications that derive new entities from the knowledge-reality-being triad) to derive all elementary mathematical functions and relations from the above primordial elements - again, with very little justification. First, the combination of multiplication and summation produces infinite series of analytic functions, and that of multiplication and graduation produces "factorials" (facultés), or infinite products of functions (INT, 8). Then come the elements that effect a transition from series to products and vice versa. These are logarithms and exponents, which indeed turn sums into products and vice versa. Among the latter, Wronski ordains as elementary those that involve a complex power, namely trigonometric functions, which turn imaginary numbers (products of reason) into trigonometric functions (belonging to the understanding as applied to intuited sense data, namely lines in circles) (INT, 12 ff.).

Once we've established the above elements, we can start relating them. First, if we study entities that depend on graduation (continuous functions) under the influence of summation (or, more appropriately, subtraction), we're dealing with the theory of differences and differentials (INT, $31 \mathrm{ff}$.). If we try to do it the other way round, we're supposed to get finite and infinitesimal analysis in terms of finite or infinitesimal powers rather than differences, which Wronski termed "grads" and "gradules" (INT, $47 \mathrm{ff}$.). This last construction was left unexplored in Wronski's later published work. When summation and graduation influence each other equally, we get the interaction between numbers as sums of units and numbers as broken into prime factors, that is, number theory and congruences (INT, 62 ff.). Finally, to bring everything together, comes the fundamental identity between the two sides: the theory that equates series and infinite products. The universal law (3) guarantees this identity (INT, $71 \mathrm{ff}$.).

There are many elements in this structure that seem forced, arbitrary, and unexplained. Wronski claimed that he discovered the law of creation, which seems to be influenced by mystical models such as the Cabalistic sephiroth (Warrain 1925), before he ventured into his mathematical discoveries (REFII, 554-555). Trying to force mathematics into the given structure of the law of creation may explain why this forcing appears so arbitrary. On the other hand, going over Wronski's work shows quite clearly a reciprocal relation where mathematics, being a privileged model of the law of creation and serving as evidence for its validity, had an important role in shaping its structure. Wronski's inclination toward esotericism can also explain why he left some of his fundamental articulations of the mathematical law of creation without justification, seemingly arbitrary and forced.

Either way, I find it hard to give a detailed charitable interpretation to Wronski's mathematical application of the law of creation. What I would like to bring up is the general framework. On the one hand we have summation, which relates to the finitist and additive empirical experience of being under the faculty understanding; on the other hand we have graduation, which is associated with knowledge, that is with the creation of an infinitary, continuous frame of reference beyond empirical experience by the faculty of reason. Together they interact in different ways so as to compose various different components of mathematics into an integral whole.

The point of this system is to put mathematics into an inherent order that exhausts mathematical entities and concepts, and gives an ontological structure to their various relations. Given such a structure, a mathematical proof would not be just a combination of correct steps, but 
an argument that respects this ontological structure and follows it.

The above law of creation belongs to the level of what Wronski calls theory. Theory studies the finite interactions of elements under the law of creation. From the point of view of theory, empirical experience and reason present magnitudes (finite and infinite respectively); the understanding then puts these magnitudes together into finite combinations, yielding analytic expressions for mathematical entities.

But at this level, formulas needn't be evaluated, and needn't be identified with their value. At this level, if two mathematical entities are to be equated, theory must guarantee that one is indeed derivable from the other, but not necessarily calculate how. Moreover, at this level of theory, mathematical entities must be finitely constructed from elementary entities, and not just determined according to their relation with other entities. In the critique of Lagrange, for example, the coefficients of the Taylor series must first be grounded in terms of theory, that is, in terms of formulas derived by finite construction from mathematical elements (including reason's infinitesimals).

To understand the scope of Wronski's notion of theory, consider the following argument, which concerns the nature of the roots of a polynomial equation of degree $k$. Wronski used some sort of idiosyncratic (but not senseless) infinitesimal analysis to show that each root is of the form $\left(1+\frac{N}{\infty}\right)^{M \infty}$, where the infinity here is actually a product of k-1 infinite quantities. He further showed that the terms of the form $\sqrt[r_{k-1}]{\left(\sqrt[r_{k-2}]{\left(\ldots \sqrt[r_{1}]{n_{1}}+\ldots n_{k-2}\right)}+n_{k-1}\right)}$ also have an identical infinitary representation. Therefore, he concluded, roots of polynomials of degree $k$ have the latter form (INT, 85-95; Servois 1814, 163-166 offers a good analysis and points out the obvious logical error in the argument: that two elements have the same structure does not mean that they must be equal).

But Wronski emphasized that this analysis concerns only the theoretical form, or nature, and not the value of the roots. In Wronski's system, this argument works because it expresses roots of polynomials in terms of the elementary quantities and operations of mathematical theory (numbers, infinities, sums, products, and powers). In the realm of theory, infinite quantities are perfectly legitimate, even though they're absent from empirical experience and cannot lead to the computation of concrete values. These are simply classificatory schemes for analyzing mathematical quantities and legitimating their comparison.

Wronski claimed that this kind of analysis sets him apart from Euler and Bezout, who simply postulated their conjectured forms of roots in order to solve fourth degree equations (REFII, cxlicxlii). Servois (1814), however, one of Wronski's most harsh, yet attentive critics, rejected the whole procedure. Indeed, it's hard to figure out rigorously what moves are allowed at this level of theoretical analysis of mathematical form and how one would avoid contradictions (working with different kinds of infinities allows some freedom so as to bypass contradictions, but this risks becoming an arbitrary post-hoc rationalization). Instead of trying to force rigor on this kind of analysis, I prefer to see it as capturing a certain qualitative or semi-formal conjectural reasoning that is nevertheless part and parcel of mathematical practice - a level of reasoning that motivates mathematicians into establishing and exploring various conjectures.

\section{Wronski's Techne}


The theoretical system of mathematical elements and relations covered above depends on the finite collection of components captured by the schematic law of creation (REFI, 13). But such a limitation cannot apply to a world whose absolute principle includes the free spontaneity of creative reason, even if it is subject to a unique absolute law. So there must be more to mathematics.

In fact, this argument applies not only to mathematics, but to truth in general. Since truth is in the relations between the spontaneous creative forms of knowledge and the given inert being, truth cannot be exhausted by experienced being, and is found in creative reason as well (PROD, 35). Wronski then goes one step further beyond this transcendental observation, and draws the conclusion that "the object of philosophy is the CREATION OF THE TRUTH, and not, as is still universally believed, KNOWLEDGE OF created TRUTHS - a knowledge which is the proper object of the SCIENCES alone" (REFII, 517; see also Warrain 1933-38, vol. 1, 91-92). As a result, Wronski's philosophical project is one of self creation (PROL, 117), and mathematics in particular is cast as the creative accomplishment of nature (REFII, 530).

In order to create truth beyond the givens of theory, one must leave the bounds of finite combinations of quantities in the understanding, and act with an aim in mind, that is, exercise a power that Wronski associates with the Kantian faculty of the will. In mathematics, this aim is to evaluate and universalize all mathematical entities under the form of series. This universalization is not something given to be discovered, but a project that man must implement. This is the transition from theory to what Wronski calls techne (technie in the original French, but referring to the Greek techne): from given individual mathematical elements to a universal generation of quantities (INT, $4 b)$.

Note that the opposition between the finite and infinite in the context of techne is different from the one in the context of theory. Indeed, in the context of theory, continuous analytic functions were on the side of continuous (and thus infinitary) graduation, and series on the side of discrete (finitary) summation. But here, from the point of view of techne, analytic functions are finite combinations of elementary entities and operations (sums, product, exponents, logarithms...) in the understandings, whereas indefinite sums require the open ended faculty of the will, which applies the power of reason to apply infinity to the finite world of intuited sense perception by going on and on without end. Indeed, finite combinations of elementary functions can only produce the small class of theoretical (or analytic) functions, whereas infinite series and products are seen as a universal mechanism that can capture all quantities (the context here is that of functional and differential equations, whose solutions may not be representable as finite combinations of elementary functions, but can be expressed in terms of series or products). Infinite series thus express the constructive will that is operative in techne (REFIII, 14-15; INT, 172-173). ${ }^{9}$

But techne faces an inherent tension between universality and evaluation. Universal formulas, such as the Taylor series or the universal formula (3), won't always converge. This does not mean that the universality of techne should be sacrificed. Indeed, in the Eulerian tradition where Wronski works, series expansions represent functions even where there is no convergence (Jahnke 1990, 198; Ferraro 2007). This is so, because the coefficients of the series are determined by the expanded function independently of convergence (as in Lagrange's construction (1)), and according to the dominant view at the time, this determination was unique (INT, 230; TECI, 93). ${ }^{10}$ This co-

9 This finality is already present, to some extent, in the theoretical relative translation of series into products and vice versa, which is part of the law of creation (see also INT, 82); but the finality of techne is expressed in active construction (INT, 243 n.).

10 This assumption, still held by Lagrange (1797, 37), was contested by Cauchy's example of exp(-1/ $\left.x^{2}\right)$, which, 
determination of functions and their expansion coefficients, which is not reducible to computable values, fits Wronski's framework, where theoretically represented analytic functions and technically generated series are different kinds of entities belonging to the different faculties of understanding and will (LAG, 58-59).

All in all, for Wronski

Series, in their divergent state, which is their most frequent state, are only pure methods of universal generation, without a real creation or production of the corresponding individual quantity. As for the state of convergence, where series effectively present such real creation of an individual quantity, there it is only a measure of the VALUE of the quantities, that is, the determination of their existence with respect to a unit taken as a term of comparison, and not the determination of the NATURE itself. (REFI, 261)

Indeed, the nature of mathematical entities is captured by finite combinations of theory's mathematical elements. In order to bridge the gap between the nature (analytic expressions), universal generation (formal series), and value (convergent series representation) of mathematical entities, Wronski devotes many pages to more or less well founded efforts that would either render divergent series convergent, or produce expansions that are guaranteed to converge (TECI, $265 \mathrm{ff}$; TECII, $299 \mathrm{ff}$; the main tools here are various transformations of the function to be expanded, changes of variable, and clever choices of the functions in terms of which one expands the given function - but this does not amount to a once and for all recipe to guarantee convergence), or provide privileged expansions that do somehow express the nature of the expanded function. ${ }^{11}$

\section{Wronski's Proof of His Universal Law}

In order to better understand Wronski's conceptual architecture, let's take as our guide Wronski's proof of his universal law (3). This proof would be considered valid in eighteenth century terms, but more suspect according to the standards developed by the mid nineteenth century. A classical analytic proof with an error term was devised by Charles Lagrange (1884a, 1884b) and a very different and modern, functional analytic proof by no less than Banach (1939). Based on the fundamental division in Wronski's philosophy, we will split the discussion into two parts, the first will concern the "theoretical" aspect of the proof, and the second the "technical" part.

\subsection{The Theoretical Part}

The starting point of the proof of the universal law (3) is the simple additive definition of a difference operator:

$$
F(x+\zeta)=F(x)+\Delta F(x) \text {. }
$$

around zero, has the same series expansion as the constant function zero. But Cauchy's view was not universally endorsed. Poisson rejected it by allowing the order of the derivative to increase as $x$ decreases (Grattan-Ginness 1990, 735-736), Martin Ohm rejected it because the derivatives at zero were not well defined in the complex plane, and Pringsheim and du Bois-Reymond later explained why, in the framework of eighteenth century algebraic analysis, the derivatives at zero of this function were not well defined (Jahnke 1990, 302-306).

11 One of the ways to induce convergence, according to Wronski, is to expand functions in terms of powers of $(x-a) /(n+x)$. Here convergence is supposedly guaranteed a-prioi by the fact that this kind of function that involves summation and multiplication "serves as transitory between the finite algorithm of summation (the basis of techne) and the infinite algorithm of graduation (the basis of theory)" (TECII, 528). This example shows how dubious theoretical considerations find their way into Wronski's mathematical statements. 
Reiterating this definition, we get

$F(x+2 \zeta)=F(x+\zeta)+\Delta F(x+\zeta)=F(x)+2 \Delta F(x)+\Delta^{2} F(x)$

and subsequently

$F(x+\mu \zeta)=F(x)+\frac{\mu}{1} \Delta F(x)+\frac{\mu(\mu-1)}{1 \cdot 2} \Delta^{2} F(x)+\frac{\mu(\mu-1)(\mu-2)}{1 \cdot 2 \cdot 3} \Delta^{3} F(x)+\ldots$.

Note that this is a finite sum with $\mu+1$ terms. Substituting $\mu=i / \zeta$ we get

$F(x+i)=F(x)+\frac{i}{1} \frac{\Delta F(x)}{\zeta}+\frac{i(i-\zeta)}{1 \cdot 2} \frac{\Delta^{2} F(x)}{\zeta^{2}}+\frac{i(i-\zeta)(i-2 \zeta)}{1 \cdot 2 \cdot 3} \frac{\Delta^{3} F(x)}{\zeta^{3}}+\ldots$.

Now, substituting the infinitesimal $d x$ for $\zeta$, we obtain the standard Taylor formula

$F(x+i)=F(x)+\frac{i}{1} \frac{d F(x)}{d x}+\frac{i^{2}}{1 \cdot 2} \frac{d^{2} F(x)}{d x^{2}}+\frac{i^{3}}{1 \cdot 2 \cdot 3} \frac{d^{3} F(x)}{d x^{3}}+\ldots$

If we replace the fixed difference $\zeta$ by a non constant difference, claims Wronski, we can tailormake our differences so as to obtain functions other than powers of $i$ in the expansion. This move involves some hand waving, as Wronski only shows this for the case of Paoli's theorem, where $i^{k}$ are replaced by some $\varphi(i)^{k}$ (TECI, 9-12).

What is the difference between this proof and Lagrange's construction, which opened this paper and which Wronski so vehemently criticized? Here we apply the elementary operation of subtraction to the function $F$. This operation (the opposite of addition) lies at the foundation of the structure of mathematics as expressed by the law of creation above. Lagrange's move, on the other hand, was completely arbitrary in Wronski's terms: dividing by the variable $i$. While formally correct, it's an accidental move, not universally applicable (indeed, occasionally a fractional power of $i$ must be used instead). As such, it cannot be assumed to provide the foundation of a mathematical theory. Because Lagrange failed to respect the ontological structure of mathematics, all he got, according to Wronski, was a partial formal theory, rather than an essential universal law.

Moreover, Wronski's move from a finite to an infinite series is made by explicitly substituting an infinitesimal for a variable. Lagrange, on the other hand, suppresses the infinitary step of his argument by an indefinite repetition, which generates ever longer finite sums, but no infinite sum as such. For Wronski it would be impossible to generate an infinite series without introducing an actual infinite quantity constituted by reason. For him, only infinity can yield infinity, and this cannot be covered over by some finitist maneuver. This is precisely the transcendent element that must be introduced into mathematics if we are to go beyond the realm of sensible finite sums. Lagrange's error here is the suppression of an ontologically necessary element: infinity.

The philosophical meaning of the universal law is in its identification of the two components: on the one hand we have a continuous function which is therefore on the side of infinitary graduation, but is finitely composed of elementary mathematical entities; on the other hand we have a summation of discrete quantities, but a summation that proceeds indefinitely. The law of creation mediates between the two sides in various ways (starting with the neutralization of summation and graduation by multiplication discussed above, and building from there; INT, 71-76; TECI, 19-20). But here, in the universal law, we finally obtain a full identification of these distinct mathematical elements: they are not only transformable to each other, they are different manifestations of infinity - the infinity inherent in graduation, and the infinity of the number of terms in the series (NOM, 126-127; REFI 11; TECI, 12; TECII, 79). ${ }^{12}$

12 In Wronski's terms, the former expressibility is teleological, whereas the latter identification is universal. I can't go into details here, but they can be gleaned from LOI 73-75; REFI 74, 258-259, and in the context of number 
The above argument is taken as a proof that the function $F$ can be expressed as a sum of any functions whatsoever, as in (3). But we must reiterate again that Wronski is only proving here what he terms the possibility of the representation, that is, its legitimacy as comparing entities which are opposed in terms of his law of creation: infinite-graduation-reason and finite-summationunderstanding. It is as much a philosophical argument about manifestations of elementary concepts as a mathematical derivation.

\subsection{The Technical Part}

The above proof does not legitimate the universal law as a tool for the actual evaluation of functions. Like the coefficients in the series expansion with non integer powers (section 3.1), in practice this form of expression may degenerate into something null or require indeterminate $(0 / 0)$ or infinite coefficients to pull together. The series expansion has a theoretical and philosophical meaning, as it expresses relations between mathematical entities, but it needn't necessarily reflect the essence of the function (its construction from elementary entities) or yield actual evaluations.

Having legitimated the theoretical possibility of the equality, one can apply reiterations to evaluate and reconstruct the nature of the coefficients. For that purpose we must apply an act of will that mediates between products of reason (such as the infinitary graduation of a continuous function) and magnitudes that the understanding can actually contain as magnitudes (such as the finite terms of a series). It is at this point that Lagrange's indefinite reiteration (2) and the formal definition of derivatives as coefficients in a series find their place in Wronski's system (INT, $224 \mathrm{ff}$.; TECI, 26 ff., 68; TECII, 75-78). Lagrange's move would have been legitimate, if it were set in the context of techne rather than theory (INT, 229). ${ }^{13}$

However, Lagrange's iterative derivation opens an indefinite process, which only constructs the first coefficients that are actually developed. One would need an inductive generalization to find the general form of coefficients (TECI, 55). Moreover, Wronski complains that such inductive generalizations often extend results from integer values of parameters to non-integer values without justification, as happens in some attempted proofs of the binomial theorem and in Laplace's derivations of generating function identities (LAP, 14-15, 53-55). ${ }^{14}$ On the other hand, if we simply posit a general formula for coefficients, and then substitute it into the equation to see that it indeed works (as, for example, in van der Monde's solution of systems of linear equations, according to TECII, 11; REFI, 316), then, according to Wronski, all we do is verification, not proof. We see here that a proof, for Wronski, must reflect an ontological structure, rather than simply string correct manipulations in sequence.

So, what alternative is there to unfounded induction and post-hoc verification? Let's observe Wronski's technical proof of his universal law. Following TECI (224 ff.), we begin with the formula (3) whose possibility we've already established:

$F=A_{0}+A_{1} \Omega_{1}+A_{2} \Omega_{2}+\ldots$.

Evaluating all functions at the point around which we expand (say, 0 ), we obtain

theory in REFI 78-82.

13 Wronski insists, however, that Lagrange skips a crucial step by assuming the form $F(x)+i P(x, i)$, rather than the more general form $F(x)+P(x, i)$, where $P(x, i)$ is then comparable to the function $i$ because they are both null at 0 (TECI, 54 n.).

14 This still happens today as well, for example in the so called "replica trick" used in calculations related to spin glasses. 
$A_{0}=F(0)-A_{1} \Omega_{1}(0)-A_{2} \Omega_{2}(0)-\ldots$.

Now, to get rid of $A_{0}$, we apply a difference operator $\Delta$ to the equation: we evaluate both sides at two values of the variable (say 0 and $d$ ), subtract the results, and equate them. We obtain $\Delta F=A_{1} \Delta \Omega_{1}+A_{2} \Delta \Omega_{2}+\ldots$

(note that if we take an infinitesimal difference, we assume the validity of term-by-term derivation).

Dividing by $\Delta \Omega_{1}$ we get:

$$
F(1)=A_{1}+A_{2} \Omega(1)_{2}+A_{3} \Omega(1)_{3}+\ldots,
$$

where we use the recursive definitions

$$
\Omega(n)_{k}=\frac{\Delta \Omega(n-1)_{k}}{\Delta \Omega(n-1)_{n}}, \Omega(0)_{k}=\Omega_{k} ; F(n)=\frac{\Delta F(n-1)}{\Delta \Omega(n-1)_{n}}, F(0)=F .
$$

We can now calculate, as above,

$$
A_{1}=F(1)(0)-A_{2} \Omega(1)_{2}(0)-A_{3} \Omega(1)_{3}(0)-\ldots \text {, }
$$

And, again, taking a difference operator and dividing, get $F(2)=A_{2}+A_{3} \Omega(2)_{3}+A_{4} \Omega(2)_{4}+\ldots$.

Continuing in this manner, we have for any positive integer $\mu$

(5) $A_{\mu}=F(\mu)(0)-A_{\mu+1} \Omega(\mu)_{\mu+1}(0)-A_{\mu+2} \Omega(\mu)_{\mu+2}(0)-\ldots$.

Note that up to this point, we express a coefficient by the infinity of following coefficients, which means that we need to know the coefficients in order to calculate them. So Wronski's next step is to eliminate $A_{\mu+1}$ by substituting its own formula, as given by (5) with $\mu+1$ substituted for $\mu$, into the identity (5) itself. We find (I omit the parentheses designating the evaluation at zero)

$$
\begin{aligned}
& A_{\mu}=F(\mu)-\Omega(\mu)_{\mu+1} F(\mu+1)-A_{\mu+2}\left(\Omega(\mu)_{\mu+2}-\Omega(\mu)_{\mu+1} \Omega(\mu+1)_{\mu+2}\right) \\
& -A_{\mu+3}\left(\Omega(\mu)_{\mu+3}-\Omega(\mu)_{\mu+1} \Omega(\mu+1)_{\mu+3}\right)-\ldots
\end{aligned} .
$$

If we continue substituting for $A_{\mu+k}$ 's indefinitely, we get

$$
\begin{aligned}
& A_{\mu}=F(\mu)+\Psi(\mu)_{1} F(\mu+1)+\Psi(\mu)_{2} F(\mu+2)+\ldots, \text { where } \\
& \Psi(\mu)_{k}=-\left(\Omega(\mu)_{\mu+k}+\Psi(\mu)_{1} \Omega(\mu+1)_{\mu+k}+\ldots+\Psi(\mu)_{k-1} \Omega(\mu+k-1)_{\mu+k}\right) .
\end{aligned}
$$

So we can now express each coefficient in terms of values of $F$ and $\Omega$ alone. The final part of the proof, which I will not survey here, uses difference of quotient formulas (analogous to differential of quotient formulas) and elementary but clever and non-transparent manipulations of combinatorial sums (determinants) in order to derive the formulas:

$$
\begin{aligned}
& \left.F(n)=\frac{X\left[\Delta^{\delta_{0}} \Omega_{0}, \Delta^{\delta_{1}} \Omega_{1}, \ldots, \Delta^{\delta_{n-1}} \Omega_{n-1}, \Delta^{\delta_{n}} F\right]}{X\left[\Delta^{\delta_{0}} \Omega_{0}, \Delta^{\delta_{1}} \Omega_{1}, \ldots, \Delta^{\delta_{n-1}} \Omega_{n-1}, \Delta^{\delta_{n}} \Omega_{n}\right]}\right] \text { and } \\
& \left.\Omega(k)_{n}=\frac{X\left[\Delta^{\delta_{0}} \Omega_{0}, \Delta^{\delta_{1}} \Omega_{1}, \ldots, \Delta^{\delta_{n-1}} \Omega_{n-1}, \Delta^{\delta_{n}} \Omega_{k}\right.}{X\left[\Delta^{\delta_{0}} \Omega_{0}, \Delta^{\delta_{1}} \Omega_{1}, \ldots, \Delta^{\delta_{n-1}} \Omega_{n-1}, \Delta^{\delta_{n}} \Omega_{n}\right.}\right],
\end{aligned}
$$

where $\delta$ is a permutation of $0,1, \ldots, n$, and the operator $X$ (originally represented by the Hebrew letter Shin), like a modern determinant, sums the expression inside the square brackets for all permutations multiplied by their signs.

This proof is analytic, in that it derives the formula for the coefficients $A_{\mu}$ from elementary arithmetic identities, rather than simply positing it and verifying by substitution. Moreover, Wronski emphasizes that this derivation is not the kind of uncertain induction attributed to Lagrange. He explains that his move reiterates the substitution of a formula that's already given "in the very 
nature of this algorithmic construction," rather than an inductive generalization of the initial cases that spring up as we go along with Lagrange's arbitrary reiteration (TECI, 228). I take this to mean that the coefficients $A_{\mu}$ in Wronski's argument are derived from repeated applications of the very difference operator that established their very possibility in the proof of the theoretical part of the universal law. Wronski's proof reconstructs their necessary composition from $F$ and the $\Omega$ 's by an inductive reiteration of the maneuvers that justify their possibility; it does not establish them by formal induction alone.

But even if we accept this explanation, it is hard to deny that the $\Psi$ 's are constructed inductively in a manner analogous to that of Lagrange's coefficients, by generalizing the form of an iterated substitution. Unfortunately, Wronski does not supply an explicit criterion for acceptable vs. unacceptable induction, which would render his maneuver legitimate as opposed to that of Lagrange. One may conjecture that the key difference may be that $\Psi$ is eventually eliminated, but my best guess is that Wronski's induction is legitimate because it builds on maneuvers that follow from the ontological derivation of the universal law, rather than Lagrange's arbitrary starting point.

A point that would raise objections for us moderns is the assumption of convergence implicit in allowing the indefinite substitution of the $A_{\mu+k}$. Wronski and his contemporaries did not raise this objection as such, but, on the other hand, they did not ignore the possibility of infinite and undetermined (0/0) coefficients (TECI, $243 \mathrm{ff}$.). Since for Wronski such quantities were legitimate elementary mathematical entities, he agrees that while they do not allow us to calculate values, they are still philosophically meaningful for the investigation of the nature of the quantities under review. The transformation of such series into convergent series or products that can be evaluated then becomes a major theme in Wronski's work on techne.

\section{Conclusion}

Wronski saw his mathematical architecture as a solution to the contemporary state of confusion in mathematics:

no link, philosophical or mathematical exists between the different branches. ... Their respective results could not ... be derived from each other. ... It is this disorder that renders the results in question FRAGMENTARY. ... In the scientific consideration of foundations reigns the greatest disorder in mathematics: the same proposition is proved in a thousand different ways to the point that ... reproducing known results has become the almost exclusive task of mathematicians. (NOM, 106-108)

This verdict is indeed exaggerated, but not without echo among other leading mathematicians. Lagrange $(1882,368)$ for example, expressed his concern in a letter to d'Alembert from 1781 that mathematics has exhausted its capacity for new results, and will soon end up like Arabic philology.

To resolve this problem, Wronski constructed an elaborate philosophical system. In his approach to the foundations of mathematics, I'd like to highlight the following aspects:

- All mathematical entities (like the entities of other sciences and practices) must be organized in a structure that reflects a universal ontology, namely the law of creation $(\S 5)$.

- This structure emerges from the unity of the absolute, bifurcates in various ways, and eventually converges to a final identity, expressed mathematically by the universal law (3). Within this structure, mathematics is a closed, exhaustible system $(\S 4, \S 6)$. Since it is seen as all but exhausted, the scientific effort should be to systematize it philosophically. 
- Within this structure, infinitary elements take up foundational roles; mathematics is one expression (among many others) of the interrelations between the infinity of constitutive reason (knowledge) and the finitude of experience bound understanding (being) $(\S 5, \S 6)$.

- A mathematical entity cannot be reduced to its value; its composition from the elements organized by the law of creation determines its nature. That a value turns out to be a zero or infinity does not mean that the corresponding entity is absent or a-mathematical $(\S 3.1, \S 3.3)$.

- Mathematical reasoning should not just be a string of formally correct moves. It must be legitimated by the structure that organizes mathematical entities and respect its internal hierarchy $(\S 3.2, \S 7)$.

For Wronski, only this approach was philosophically true. Only by following through this project could one extract from mathematical reasoning a complete solution to all mathematical problems, rather than an assortment of partial results. But Wronski's attempt was rejected. Reflecting on Wronski's architecture of knowledge, Servois reacted in a way that many modern mathematicians would sympathize with, and which seems to have reflected the general state of mind. He compared Wronski's attempt at reducing mathematics to a single foundation with the attempts of scholastics and Port-Royal logicians to reduce mathematics to their respective narrow logical systems. Moreover, Servois found Wronski's notion of infinity self contradictory (based on Kant's rejection of the constitutive infinite in favor of the regulative indefinite), and stipulated that "there are no fundamental laws except for definitions, which are not objects to be discovered" (Servois 1814, 168-169).

We see here that Wronski's conception of proofs and foundations had little in common with the mathematical community that he tried to address, and his approach was aggressively and promptly marginalized. There were good reasons for this marginalization. Wronski's writing was hard to follow, and relied on a philosophical system that was only exposed in France after he had no longer been taken seriously as a mathematician. He confronted aggressively the leading members of the small community who could deal with higher mathematics, but failed to take into account his weak position as an outsider. His claims were even more megalomaniac and more prone to over generalization than those of his contemporaries (who were themselves already prone to self congratulation and over-generalized claims). Furthermore, the esoteric dimension of his workwithholding proofs and the intimate essence of his philosophy - made him look like a quack.

But even if Wronski had been better at communicating with those in his environment, it is not likely that the mathematical community would have embraced his approach. In Europe, at least since late antiquity, mathematics had the secure core of Euclidean geometry and arithmetic, and beyond it more or less contested fringes. But since the sixteenth century these contested fringes have grown more dominant, and controversies began to emerge over philosophy, mathematics and authority, which threatened to shake the image of mathematics as an indubitably sound foundation. The mathematical elite of post-revolutionary France, organized around the Académie des Sciences, needed to restore a core of consensus in order to maintain its position as the curator of truth. Finitist-formalist tendencies and an applied focus went toward the establishment of such a core. Wronski, on the other hand, tried to bring back philosophical debates in a way that was alien to the pragmatic mainstream and depended on highly unstable philosophical and mathematical arguments.

Moreover, Wronski's attempt to organize all mathematics into a finite theoretical framework and to subsume all results under a universal ur-result were inhibitive in a period characterized by new constructions and approaches. Wronski's supposedly comprehensive classification of 
mathematical elements and methods risked preventing the creation of new mathematical entities and methods (for instance, Wronski's universal law did not find use in the context trigonometric series, as his entire approach depended on term-by-term derivation of series, which fails for the most useful trigonometric series). ${ }^{15}$ This might have turned mathematics into a dying science, exactly as Lagrange had feared. It is against these circumstances that the styles, projects, and priorities of the successful mainstream mathematicians of the time can be appreciated.

Beyond its role as a vignette of the contingency of early nineteenth century mathematical developments, I'd like to suggest two directions for integrating the study of Wronski with other directions of research. The first would place Wronski in the tradition of foundational debates in a way that might better historicize it. The seventeenth-eighteenth century controversies concerning the foundations of mathematics (around infinitesimals) are well known, and so are the debates of the early twentieth century. If we wish to understand the latter, not only as an internal reaction to a supposed foundational crisis, but also as part of a continuous tradition of collisions and alliances between philosophy and mathematics, we need to look into the nineteenth century German idealist and Neo-Kantian philosophy of mathematics as well (e.g. Fichte, Hegel, Fries, Hermann Cohen).

In some ways, the foundationalist movements of the early twentieth century were in line with Wronski's attempt at contesting the autonomy of mathematics in order to set it within a proper philosophical framework. The intuitionist reform threatened to do away with entire branches of mathematics; the formalist empty structures undermined ways of doing mathematics that depended on geometric intuition (e.g. the Italian school of algebraic geometry); the logicists burdened mathematics with unmanageable type and order theories - all for the benefit of placing mathematics in what was seen to be its proper place within the larger realm of knowledge.

These foundational attempts show that the value of mathematics lies not just in its scientific use, but also in its philosophical and cultural positioning. Wronski's attempt can be used to remind us of this fact, and help us trace intellectual courses leading to the modern foundational programs. Indeed, one shouldn't rule out a priori the possibility that Wronski's Kantian and constructive emphases (as opposed to his endorsement of actual infinities) may have had some indirect role in the formation of intuitionist and Neo-Kantian conceptions (Hermann Cohen's philosophy of mathematics, for example, has a lot in common with Wronski's), as despite his marginalization by his contemporaries, Wronski did attract some attention toward the end of the nineteenth century.

The other suggestion is to read Wronski against his own explicit grain: instead of as an ontologist of mathematics, we could read Wronski as a philosopher of mathematical practice. Indeed, Wronski's division of mathematical knowledge into various aspects, based on different faculties, did reflect different mathematical practices: "theoretical" arguments based on informal analogies and generalizations, manipulations of "ideal" entities, formal vs. content based manipulations, and infinitary vs. finitary constructions. Much of this was and is highly relevant to mathematical practice, which depends on half baked intuitive conjectures, formal analogies and reasoning, reification of infinities and ideal solutions (such as complex solutions of polynomial equations and $L^{2}$ solutions of differential equations), analytic heuristic approaches, and synthetic formal proofs.

15 Wronski focused on expansions in terms of rational and algebraic functions, rather than trigonometric functions. He did, however, introduce "higher trigonometric functions." These functions are sine and cosine like

functions formed by choosing n-th roots of unity $\rho$ and $\sigma$, and extracting from the power series expansion of $e^{\rho x}$ the series of those terms which are real multiples of $\sigma$. These functions were discussed as tools for analyzing celestial mechanics, but never explicitly as an alternative to Fourier series. 
Wronski tried to provide each faculty or practical approach with its proper place in a larger system that sought to integrate them all. But his system was not articulated clearly enough in order to help decide which kind of arguments were admissible in which domain, and it carried clear traces of cutting corners and drawing a mark around the arrow in order to maintain the illusion of a viable structure. Wronski's system, therefore, is hardly usable as a rigorous philosophical articulation. However, the broader outlines of Wronski's system of mediation and integration of different mathematical faculties may be useful for contemporary philosophers of mathematical practice, and his specific articulations may shed some light on early nineteenth century mathematical practice. 


\section{Abbreviations of cited publications by Wronski}

Wronski's works and manuscripts are all freely available online at the Wielkopolska Biblioteka Cyfrowa website. Here is a chronological list of their titles and the abbreviations used in this paper.

PHI = Philosophie Critique, Fondée sur le Premier Principe du Savoir Humain (1803)

INT = Introduction à la Philosophie des Mathématiques et Technie l'Algorithmie (1811)

$\mathrm{LAG}=$ Réfutation de la Théorie des Fonctions Analytiques de Lagrange (1812)

$\mathrm{INF}=$ Philosophie de l'Infini Contenant des Contre-Réflexions et des Réflexions sur la Métaphysique du Calcul Infinitésimal (1814)

TECI = Philosophie de la Technie Algorithmique, vol. I (1815)

TECII = Philosophie de la Technie Algorithmique, vol. II (1816-7)

LAP = Critique de la Théorie des Fonctions Génératrices de Laplace (1819)

PROD = Prodrome du Messianisme; Révélation des Destinées de l'Humanité (1831)

$\mathrm{PROL}=$ Le Destin de la France, de l'Allemagne et de la Russie comme Prolégomènes du Messianisme (1842-3)

REFI = Messianisme ou Réforme Absolue du Savoir Humain: Vol. I. Réforme des Mathématiques (1847)

REFII = Messianisme ou Réforme Absolue du Savoir Humain: Vol. II. Réforme de la Philosophie (1848)

REFIII = Messianisme ou Réforme Absolue du Savoir Humain: Vol. III. Résolution Générale et Définitive des Equations Algébriques de Tous les Degrés (1848)

APOD = Apodictique Messianique ou Traité du Savoir Suprême (1876)

LEG = "Philosophie ou Législature de Mathématiques." In Sept Manuscrits Inédits - Ecrits de 1803 à 1806. (1879)

$\mathrm{NOM}=$ Nomothétique Messianique ou Lois Suprêmes du Monde (1881)

LOI = Loi Téléologique du Hasard, comme Base de la Réforme du Calcul de Probabilités (1890)

\section{References}

D'Arcy, Philippe. 1970. Hoene-Wronski: Une philosophie de la création. Paris: Seghers.

Banach, Stephan. 1939. “Über das 'Loi Suprême de J. Hoene-Wroński.” Bulletin International de l'Académie Polonaise des sciences et des lettres, Ser. A, 1-10.

Carnot, Lazare. 1832. Reflexions on the Metaphysical Principles of the Infinitesimal Analysis. Oxford: J. H. Parker.

Cauchy, Augustin Louis. 2009. Cauchy's Cours d'Analyse: An Annotated Translation. New York, NY: Springer.

Dickstein, Samuel. 1896. Hoene Wroński-Jego Życie I Prace [please add an English translation of the title here: Hoene Wroński - His Life and Work]. Krakow: Nakładem Akademii Umiejętności. 
Dickstein, Samuel. 1892-1896. “Sur les Découvertes Mathématiques de Wronski.” Biblioteca Mathematica 6:48-52, 85-90; 7:9-14; 8:49-54, 85-87; 10:5-12.

Ferraro, Giovanni. 2007. "Convergence and Formal Manipulation in the Theory of Series from 1730 to 1815." Historia Mathematica 34:62-88.

Grattan-Guinness, Ivor. 1990. Convolutions in French Mathematics, 1800-1840, vol 2. Basel: Birkhäuser.

Jahnke, Hans Niels. 1990. Mathematik und Bildung in der Humboldtschen Reform. Göttingen: Vandenhoeck \& Ruprecht.

Jahnke, Hans Niels.1993. “Algebraic Analysis in Germany, 1780-1840: Some Mathematical and Philosophical Issues.” Historia Mathematica 20:265-284.

Lagrange, Charles Henri. 1884a. "Forme générale du reste dans l'expression d'une fonction au moyen d'autres fonctions." Comptes Rendus de l'Académie des Sciences de Paris 98:14221425 .

Lagrange, Charles Henri. 1884b. "Démonstration elémentaire de la loi suprême de Wronski." Mémoires Couronnés et Mémoires des Savants Etrangers Publiés par l'Académie Royale des Sciences, des Lettres et de Beaux-Arts de Belgique 47.

Lagrange, Joseph-Louis. 1797. Théorie des fonctions analytiques contenant les principes du calcul différentiel. Paris: Ecole Polytechnique.

Lagrange, Joseph-Louis. 1882. Oeuvres de Lagrange, vol. XIII. Paris: Gauthier-Villars.

Laplace, Pierre Simon. 1812. Théorie analytique des probabilités. Paris: Courcier.

Medvedev, Fyodor A. 1991. Scenes from the History of Real Functions. Basel: Birkhaeuser.

Muir, Thomas. 1882. A Treatise on the Theory of Determinants. London: Macmillan.

Murawski, Roman. 2005. "Genius or Madman? On the Life and Work of J. M. Hoene-Wroński.” In European Mathematics in the Last Centuries, edited by W. Więsław, 75-86. Wrocław: Typoscript Studio Wydawniczo-Typograficzne Andrzej Ploch.

Murawski, Roman. 2006. “The Philosophy of Hoene-Wroński.” Organon 35:144-150.

Phili, Christine. 1996. "La loi suprême de Hoëné-Wronski: La rencontre de la philosophie et des mathématiques." In Paradigms and mathematics, edited by Elena Ausejo and Mariano Ormigon, 289-308. Madrid: Siglo XXI de España Editores. 
Poisson, Siméon Denis. 1805. "Démonstration du théorême de Taylor." Correspondance sur l'Ecole Imperiale Polytechnique 1(3):52-55.

Pragacz, Piotr. 2008. "Notes on the Life and Work of Józef Maria Hoene-Wroński.” In Algebraic Cycles, Sheaves, Shtukas, and Moduli, edited by Piotr Pragacz, 1-20. Basel: Birkhäuser.

Schubring, Gert. 2005. Conflicts between Generalization, Rigor and Intuition. New York: Springer.

Séguin, Philippe. 2005. "La recherche d'un fondement absolu des mathématiques par l'Ecole combinatoire de C.F. Hindenburg (1741-1808).” Philosophia Scientiae 5:61-79.

Servois, François Joseph. 1814. "Réflexions sur les divers systèmes d'exposition des principes du calcul cifférentiel, et, en particulier, sur la doctrine des infiniment petits." Annales de Gergonne 5:141-170.

Wagner, Roy. 2014. "Wronski's Infinities.” HOPOS (History of Philosophy of Science) 4:26-61.

Warrain, Francis. 1925. L’armature métaphysique de Hoëne Wronski. Paris: Alcan.

Warrain, Francis. 1933-1938. L'oeuvre philosophique de Hoené Wronski: Textes, commentaires et critique, 3 vols. Paris: Vega.

West, Emil. 1886. Exposé des méthodes générales en mathématiques: résolution et intégration des équations, applications diverses d'Après Hoénë Wronski. Paris: Gauthier-Villars. 